\title{
Strates
}

STRATES Matériaux pour la recherche en sciences sociales

$7 \mid 1993$

Témoins du monde : Bulgarie, identités chinoises, explorer l'île de France

\section{De la ville à l'urbanisation}

de Gilles Montigny

\section{Catherine Rhein}

\section{(2) OpenEdition}

Journals

Édition électronique

URL : http://journals.openedition.org/strates/1186

DOI : 10.4000/strates. 1186

ISSN : $1777-5442$

Éditeur

Laboratoire Ladyss

Édition imprimée

Date de publication : 30 juin 1993

ISSN : 0768-8067

\section{Référence électronique}

Catherine Rhein, « De la ville à l'urbanisation », Strates [En ligne], 7 | 1993, mis en ligne le 13 décembre 2005, consulté le 08 septembre 2020. URL : http://journals.openedition.org/strates/1186 ; DOI :

https://doi.org/10.4000/strates. 1186

Ce document a été généré automatiquement le 8 septembre 2020.

Tous droits réservés 


\title{
De la ville à l'urbanisation
}

\author{
de Gilles Montigny ${ }^{1}$
}

\section{Catherine Rhein}

1 Dans ce livre qu'il faut désormais placer parmi les ouvrages de fond sur l'histoire de la recherche urbaine, Gilles Montigny offre avec beaucoup d'honnêteté et de générosité une mine de références et d'informations sur les études urbaines développées dans et autour du monde académique français entre la fin du XIX ${ }^{\mathrm{e}}$ siècle et les années 1920.

2 C'est principalement au repérage et à l'analyse du contenu de ces très nombreuses études et recherches que Gilles Montigny s'est attaché dans cet important travail de thèse. Le problème de la formation des disciplines, d'écoles et de milieux professionnels est évoqué dans les premiers chapitres et l'auteur aborde (un peu timidement) la question des relations entre le développement des études urbaines et l'intervention certes encore limitée - de l'État en matière urbaine. Enfin et surtout, Gilles Montigny ne force jamais l'interprétation des travaux qu'il évoque et sait respecter les auteurs qu'il sort de l'oubli et dont il donne à voir les œuvres, ouvrant ainsi de nouvelles et nombreuses pistes de recherche.

3 Indiquons les deux types d'apports majeurs de cet ouvrage. D'abord, il faut le dire et le redire, cet ouvrage comble un vide béant sur l'histoire de la recherche urbaine; cette méconnaissance a amené à redécouvrir deux, trois, voire quatre fois les mêmes « lois ", les mêmes processus. Marie-Claire Robic l'avait déjà démontré à propos de la théorie des lieux centraux ${ }^{2}$ et Gilles Montigny l'illustre ici, notamment à propos des quatre lois du grand statisticien social que fut Émile Levasseur, lois énoncées dans La Population Française (publiée entre 1889 et 1892): sur la force d'attraction des villes, sur les «cercles concentriques" du développement urbain, sur la relation directe entre densité et prix des terrains, enfin entre développement économique et degré d'urbanisation (p. 318). Plusieurs chapitres de l'histoire de la géographie française sont donc à revoir, voire tout simplement à réécrire.

Deuxième ensemble d'apports, celui de bibliographies portant sur des auteurs peu connus ou méconnus (notamment Paul Meuriot - pp. 363-372 - et René Maunier) et de bibliographies issues du dépouillement méthodique des travaux et notices 
bibliographiques parus dans Les Annales de Géographie et dans l'Année Sociologique (pp. 120-136).

Une critique, cependant: l'absence d'une bibliographie récapitulative des travaux sur cette histoire des études urbaines et l'insuffisance des index. Ces références sont disséminées au fil des nombreuses notes regroupées à la fin de chaque chapitre, solution peu coûteuse pour l'éditeur, calamiteuse pour le lecteur quand il a affaire à un auteur aussi méticuleux que Gilles Montigny. Seul un index des "termes importants » figure en fin d'ouvrage, là où il faudrait aussi absolument un index des lieux et un index des auteurs. Bref cet ouvrage n'a pas les annexes qu'il mérite et c'est bien dommage.

\section{NOTES}

1. De la ville à l'urbanisation, essai sur la genèse des études urbaines françaises en géographie, sociologie et statistique sociale, préf. de M. RONCAYOLO, Paris, L'Harmattan, 1992, 376 p.

2. Marie-Claire ROBIC, « Cent ans avant Christaller : une théorie des lieux centraux », L'Espace géographique, vol. XI, 1982, pp. 5-12.

\section{AUTEUR}

\section{CATHERINE RHEIN}

Chargée de recherche au CNRS (STRATES), elle poursuit des travaux sur les processus, les formes et les facteurs de la division sociale de l'espace dans l'agglomération centrale de Paris depuis les années 1920. 